\title{
Numerical results for coagulation equation with bounded kernels, particle source and removal
}

\author{
C. D. Calin, M. Shirvani \& H. J. van Roessel \\ Department of Mathematical and Statistical Sciences, \\ University of Alberta, Edmonton, Alberta, Canada
}

\begin{abstract}
Numerical approaches for the coagulation equation with source and removal terms, and kernels that are bounded independently of the particle size, are investigated. A few classes of exact solutions are provided.

Keywords: coagulation, polymers, exact solution, source, removal, numerical solution.
\end{abstract}

\section{Introduction}

There is considerable literature on the mathematical theory of coagulation, deterministic and stochastic, discrete and continuous, beginning with the pioneering work of Smoluchowski in 1917 on modelling binary coalescence of particles. For a very comprehensive survey of work up to 1970, including applications, different derivations of the equation from physical assumptions, and discrete versions of the equation, see Drake [1]. The pioneering works of Melzak [2] (on cloud formation) include some of the earliest applications of the theory, and more applications can be found in Drake [1], Lee [3], Krivitsky [4].

The presence of external particle sources, and the removal of particles from the system, however, has not received a great deal of mathematical attention, the work of Simons [5] being a notable recent exception. In Shirvani and Van Roessel [6], the discrete version with constant kernel and source terms is investigated. Laurençot [7] and Norris [8] are two comprehensive recent studies of coagulation/fragmentation for unbounded kernels (but without source and removal effects).

An example of application to the coagulation equation is in the manufacturing of aluminium alloys. Here, molten metal is kept in a holding furnace for several hours while particles of titanium diboride are added for further solidification and casting. 
During this process these foreign particles can agglomerate and be lost from the melt by attachment to the furnace walls, thus jeopardizing the desired properties of the alloy, and increasing manufacturing costs (see, e.g. Wattis et al [9]). Although there have been significant studies regarding the size distribution in molten aluminum, still not much is known about the kinetics of the coagulation in this system. This is an example of an industrial process where one may wish to increase or modify the number of particles of a particular size. The only way to achieve this would be by the introduction or removal of particles of some prescribed size to enable the coagulation process to arrive at some desired limiting state.

The equation being investigated in this paper is

$$
\begin{aligned}
\frac{\partial u}{\partial t}(x, t) & =\frac{1}{2} \int_{0}^{x} K(x-y, y, t) u(x-y, t) u(y, t) d y \\
& -u(x, t) \int_{0}^{\infty} K(x, y, t) u(y, t) d y+g(x, t)-r(x, t) u(x, t)
\end{aligned}
$$

subject to the initial condition

$$
u(x, 0)=u_{0}(x) \geq 0
$$

For a detailed description of the terms in eqn. (1) see for example Drake [1] and Melzak [2]. In this paper, the coagulation kernel $K$ is assumed to be bounded (for the precise mathematical form of these assumptions see Calin et al [10]). Bounded kernels are of fundamental importance both practically as well as theoretically (for investigating unbounded kernels, using the method of truncation). They were first investigated by Melzak [2] in the case where no source term is present.

The source $g$ is the rate of addition of new particles to the system, and $r$ determines the rate of removal of particles from the system. For physical reasons, the source and removal terms are assumed to be non-negative. None of the functions $K, g, r$ is assumed to be continuous.

In this article we provide information about the numerical approaches to the solution of $(1,2)$, and comment on how the solutions depend on factors such as the kernel $K$ or the source term $g$ (see Section 2). The two most reliable methods (collocation and adaptive power series) are described in some detail. In Section 3 we comment on explicit solutions for particular choices of $K$ and $g$ (closed-form solutions cannot be expected to exist in the general case).

\section{Numerical results}

In applications (industrial or otherwise), even the kernel $K$ may not be known analytically, and the question of finding a reliable numerical solution to $(1,2)$ becomes important. There are two distinct problems here.

One is the question of computing the values of $u(x, t)$ for a bounded, predetermined range of values $0 \leq x \leq X$ and $0 \leq t \leq T$. This is the correct setting in many industrial problems, where the physical limits $X$ on particle size and $T$ on 
reaction time arise naturally. In such cases we may, if desired, find constants $a, b, c$ such that the change of variables

$$
x=a x^{*}, \quad t=b t^{*}, \quad u=c u^{*},
$$

transforms $(1,2)$ into an analogous equation with the same $K$, but with $0 \leq$ $x^{*}, t^{*} \leq 1$ (or any other finite upper limits; the modification is in $u_{0}, g, r$ being multiplied by various constants). In other words, in this type of problem it is legitimate to confine $x$ and $t$ to a pre-determined range of values.

The other problem (which we shall not discuss here) typically involves a change of variables $t^{*}=\psi(t)$, the function $\psi$ being chosen in such a way that the entire interval $0 \leq t<\infty$ corresponds to $0 \leq t^{*}<1$; a popular choice is $t^{*}=\left[\int_{0}^{\infty}\left(u_{0}(x)-u(x, t)\right) d x\right] /\left[\int_{0}^{\infty} u_{0}(x) d x\right]$. This is most suitable for studying the long-time properties of $u(x, t)$, since $t \rightarrow \infty$ corresponds to $t^{*} \rightarrow 1^{-}$. The method, however, appears to be less reliable numerically for bounded ranges of the values of $t$. In recent years, several numerical studies have been devoted to (1), (see, e.g. Filbet and Laurençot [12], Krivitsky [4], Lee [3]).

Our numerical results are presented for $0 \leq x \leq 5$ and $0 \leq t \leq 1$ following the comments at the beginning of this section. Quite a few numerical schemes were looked at, and the two methods giving the most accurate results (when tested in the cases where exact solutions are known) were found to be the weighted residual method (collocation method) and the method of power series at successive points (the adaptive PS). A description of the methods follows.

One of the more reliable methods of obtaining numerical solutions to (1) turns out to be the use of power series. If $K$ is independent of time, and we have

$$
u(x, t)=\sum_{i=0}^{\infty} \gamma_{i}(x) t^{i}, g(x, t)=\sum_{i=0}^{\infty} \delta_{i}(x) t^{i}
$$

for some interval of values of $x$ and $t$, then substitution into (1) (again with $r=0$ ) evidently leads to $\gamma_{0}(x)=u_{0}(x)$, and for $n \geq 0$,

$$
\begin{aligned}
(n+1) \gamma_{n+1}(x)=\delta_{n}(x) & +\frac{1}{2} \sum_{i+j=n} \int_{0}^{x} K(y, x-y) \gamma_{i}(y) \gamma_{j}(x-y) d y \\
& -\sum_{i+j=n} \gamma_{i}(x) \int_{0}^{\infty} K(x, y) \gamma_{j}(y) d y
\end{aligned}
$$

The question of the convergence of the series $\sum_{i=0}^{\infty} \gamma_{i}(x) t^{i}$ is a very interesting one, not least because there is more than one sense in which the series can converge. The question of convergence and an example of its use will be discussed in Calin et al [10]. For additional comments on the method of power series expansions in terms of the small parameter $t^{*}=\psi(t)$, see Martynov and Bakanov [11]. They comment that, for certain kernels, using 10 terms in the series gave reasonable 
results only for $t^{*} x \leq 2$. This is only practical for the initial stages of the evolving spectrum. Drake [1] suggests the use of power series combined with asymptotic methods for obtaining global numerical solutions.

In using a partial sum $u(x, t) \sim \sum_{i=0}^{m} \gamma_{i}(x) t^{i}$ for relatively large times $t$, a modification is found to be useful. Let $\delta>0$ be small, and suppose we want to find the value of $u(x, t)$ at $t=n \delta$ for some large $n$. Beginning with $\gamma_{0}=u_{0}$, compute $\gamma_{1}, \ldots, \gamma_{m}$ from (3), and then obtain $u(x, \delta) \sim u^{(1)}(x)=\sum_{i=0}^{m} \gamma_{i}(x) \delta^{i}$. However, to compute $u(x, 2 \delta)$, it is better to start with a new $\gamma_{0}^{(2)}=u^{(1)}$, re-compute the corresponding $\gamma_{1}^{(2)}, \ldots, \gamma_{m}^{(2)}$ from (3), and then use $u(x, 2 \delta) \sim u^{(2)}(x)=$ $\sum_{i=0}^{m} \gamma_{i}^{(2)}(x) \delta^{i}$ (this is tantamount to computing the Taylor series at $t=\delta$, which is in turn equivalent to shifting the origin of time to $t=\delta$, and then solving the initial-value problem). Proceeding in this way, the numerical results were found to be much more precise than when a single series $\sum_{i=0}^{m} \gamma_{i}(x) t^{i}$ was used for increasingly larger values of $t$.

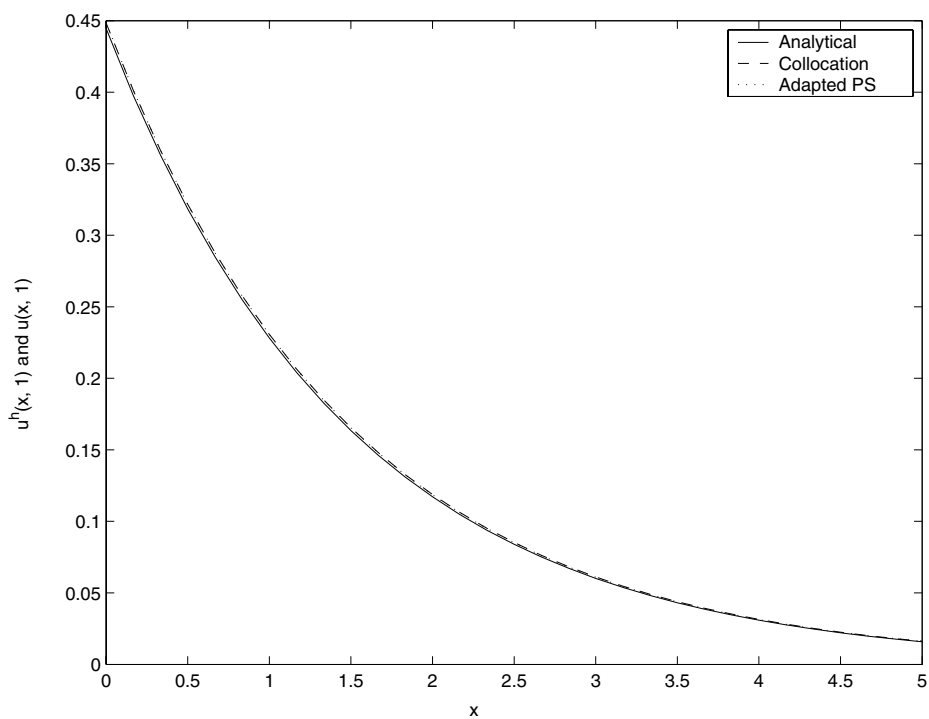

Figure 1: Plot of the numerical solution $u^{h}(x, 1)$ using the collocation and the adaptive power series methods and analytical solution $u(x, 1)$.

The version of the collocation method used here is the one suggested in Sandu [13]. Taking the collocation points to be the same as the nodal points, the integral terms of the coagulation equation were evaluated by using Gaussian numerical quadrature. Having performed the pointwise evaluation of the terms of the coagulation equation at the nodal points, the original partial integro-differential equation is transformed into a set of ordinary differential equations, where the dependent variables are at the same points. This system was then solved by the semi-implicit Euler for the time-discretization. Our experiments showed excellent 
accuracy even with piecewise-linear elements and with a small number of size bins.

Even though the collocation method requires integration only at the nodal points and seems to have good accuracy even with linear elements, computationally speaking it is an expensive method. For instance, using 31 bins yields a maximum error of $1.67 \times 10^{-3}$ with the collocation method, and a maximum error of $5 \times 10^{-3}$ for the adaptive power series method (with terms up to and including $t^{2}$ ) in the example presented below (the errors were found to be of a similar order of magnitude in other examples).

Our conclusion from repeated testing is that, for examining the qualitative behaviour of the solutions, the adaptive power series (even with as few as three terms) is quite accurate, while for more precise numerical solutions, the collocation method is preferable.

Letting $u^{h}$ denote the numerical solution, Figure 1 shows the graph of $u^{h}(x, 1)$ for the case $K=1, g=0$, and $u_{0}(x)=e^{-x}$. The exact solution $u(x, t)=$ $(1+t / 2)^{-2} e^{-2 x /(2+t)}$ is obtained in Example 3.1 (with $\eta(t)=0$ in the notation of that example).

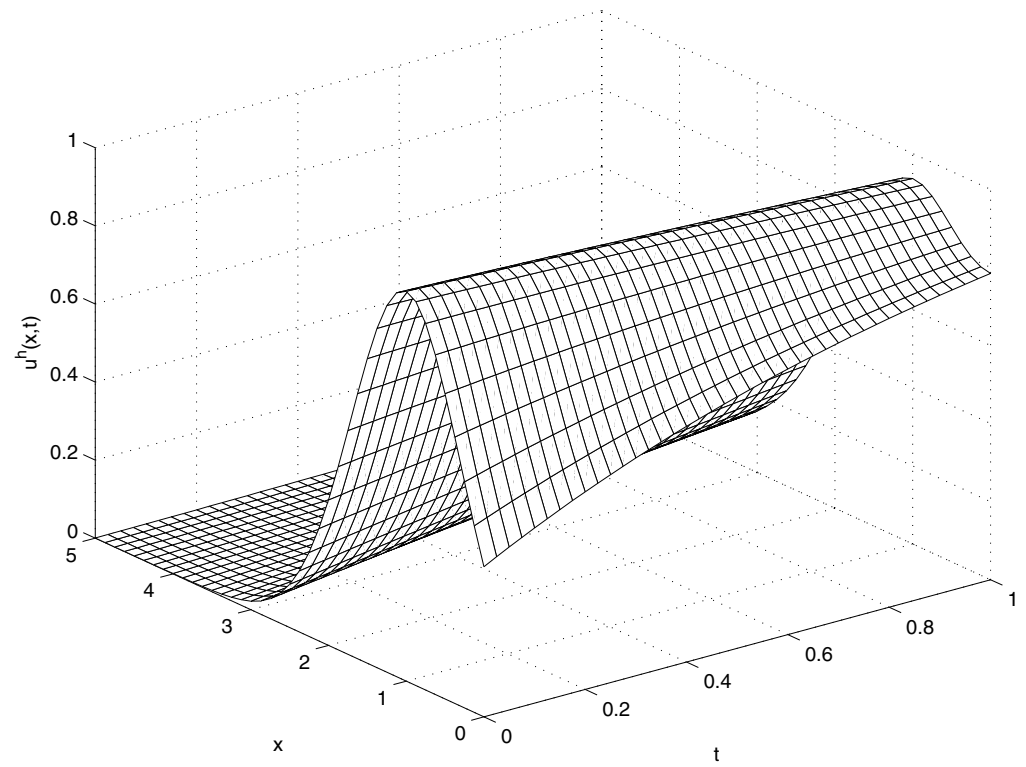

Figure 2: $K(x, y)=1 /(1+x+y), \quad g(x, t)=e^{-x}, \quad u(x, 0)=e^{-(x-1)^{2}}$.

The above picture shows the propagation of an initial global maximum through time. The adaptive power series method was used in this and subsequent graphs. Longer time periods can be investigated by a suitable change of variables as indicated earlier in this section, but result in no qualitative change in behaviour. No analytical solution is known in general for this kernel. 


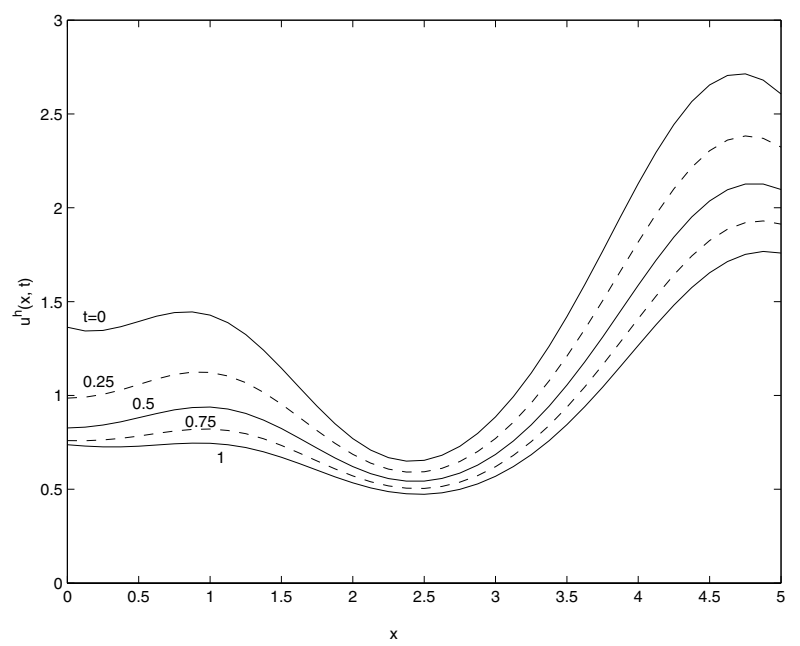

Figure 3: The graph of the numerical solution $u^{h}(x, t)$ at times $t=$ $0,0.25,0.5,0.75,1$ assuming the initial condition $u(x, 0)=e^{-\sin x}+$ $e^{-(x-1)^{2}}$ has two maxima and $K(x, y)=1 /(1+x+y), \quad g(x, t)=$ $e^{-x}$.

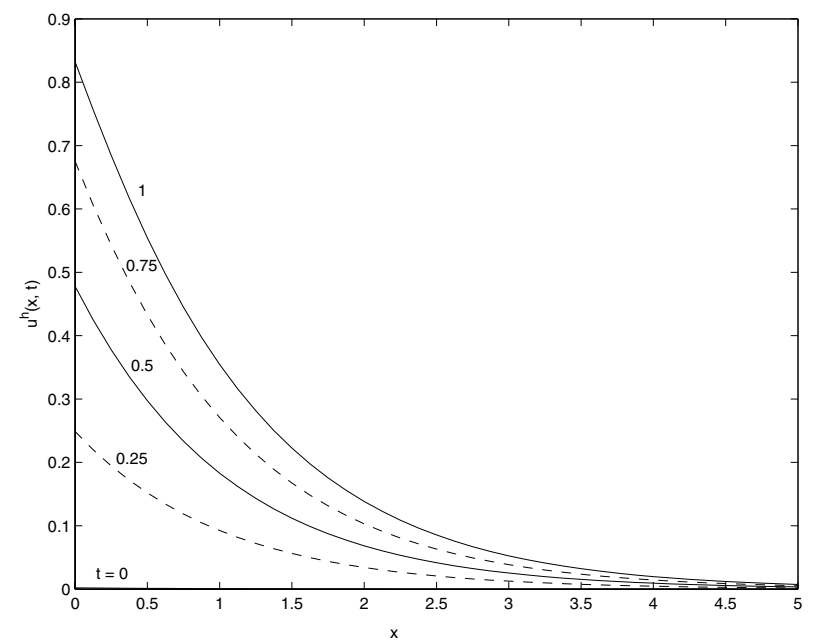

Figure 4: $K(x, y)=1 /(1+x+y), \quad g(x, t)=e^{-x}, \quad u(x, 0)=0$.

The graph in Figure 4 shows the influence of the source term on the solution. The solution increases from its initial value of $u_{0}(x)=0$. The series of graphs in Figure 4 also indicates the fact that the kernel $K$ exerts a relatively small and 
transient influence on the form of the solutions, with the initial conditions $u_{0}$ and the source term $g$ being the more dominant factors.

Our next example (Figure 5) is that of another intractable kernel, $K(x, y)=$ $e^{-\left(x^{2}+y^{2}-1\right)^{2}}=e^{-\left(r^{2}-1\right)^{2}}$ (in polar coordinates). Observe that the maxima of $K$ initially appear in the solution before being smoothed out by the coagulation process.

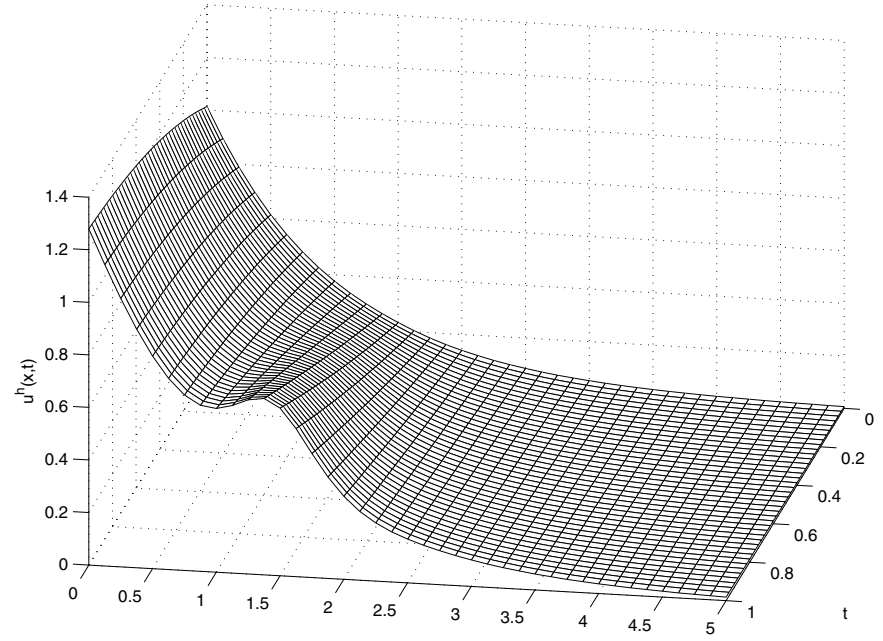

Figure 5: $K(x, y)=e^{-\left(x^{2}+y^{2}-1\right)^{2}}, \quad g(x, t)=e^{-x}, \quad u(x, 0)=e^{-x}$.

\section{A few examples of exact solutions for (1)}

In this section we look at exact solutions to (1). For more examples of exact, formal and asymptotic solutions see Calin et al [10]. It is worth mentioning that no closedform solution of (1) is known when $K=0$ outside a bounded interval (except in trivial cases).

Example 3.1 Our first example is an explicit solution to (1). Assume that $K \equiv 1$. Let $\eta(t)$ be a non-decreasing, non-negative function for all $t \geq 0$, such that $\eta(0)=0$. Set $\alpha(t)=2 /\left[2+\int_{0}^{t} e^{\eta(s)} d s\right]$. If the source term is $g(x, t)=$ $\eta^{\prime}(t) \alpha^{2}(t) e^{\eta(t)-x \alpha(t)}$ and the initial condition is $u_{0}(x)=e^{-x}$, then it can be verified by a direct substitution that $u(x, t)=\alpha^{2}(t) e^{\eta(t)-x \alpha(t)}$ is the solution of (1).

Example 3.2 The next example shows that, in general, separable, non-negative solutions cannot be expected to exist for all $x$. To see this, suppose $u(x, t)=$ $u_{0}(x) \beta(t)$ for $(x, t) \in[0, N] \times[0, \infty)$ for some $N>0$, where $\beta(0)=1$. Substi- 
tuting into (1) with $r=0$ and re-arranging, we find that

$$
\frac{g}{\beta u}=\frac{\beta^{\prime}}{\beta^{2}}+\int_{0}^{N} K(x, y) u_{0}(y) d y-\frac{1}{2} \int_{0}^{x} \frac{K(y, x-y) u_{0}(y) u_{0}(x-y)}{u_{0}(x)} d y .
$$

Now let

$$
p(N)=\inf _{x \in[0, N]}\left[\int_{0}^{N} K(x, y) u_{0}(y) d y-\frac{1}{2} \int_{0}^{x} \frac{K(y, x-y) u_{0}(y) u_{0}(x-y)}{u_{0}(x)} d y\right] .
$$

Then of course

$$
\frac{g}{\beta u} \geq \frac{\beta^{\prime}}{\beta^{2}}+p(N)
$$

If $p(N) \geq 0$, then the most general function $\beta$ for which the right-hand side of the above equation remains non-negative is

$$
\beta(t)=\frac{1}{1+p(N) t-\eta(t)}
$$

where $\eta$ is any non-decreasing function of $t$ such that $\eta(0)=0$ and $\eta(t)<1+$ $p(N) t$ for all $t$. The simplest choice is $\eta(t)=0$ for all $t$. In order for a separable non-negative solution to exist for all $x \geq 0$, we require that $\lim _{N \rightarrow \infty} p(N) \geq 0$. We do not know of any examples (even with $K=1$ ) where the above condition holds (the limit is $-\infty$ in the examples we have looked at).

\section{References}

[1] Drake, R.L., A general mathematical survey of the coagulation equation, In: G. Hidy and J. R. Brock, editors, Topics in Current Aerosol Research 3 (Part 2), Pergamon Press, 1972.

[2] Melzak, Z.A., A scalar transport equation, Trans. Amer. Math. Soc., 85, pp. 547-560, 1957.

[3] Lee, M.H., A survey of numerical solutions to the coagulation equation, $J$. Phys. A, 34, pp. 10219-10241, 2001.

[4] Krivitsky, D.S., Numerical solution of the Smoluchowski kinetic equation and asymptotics of the distribution function, J. Phys. A, 28, pp. 2025-2039, 1995.

[5] Simons, S., On the solution of the coagulation equation with a timedependent source-application to pulsed injection, J. Phys. A:Math. Gen., 31, pp. 3759-3768, 1998.

[6] Shirvani, M. \& van Roessel, H.J., Existence and uniqueness of solutions of Smoluchowski's coagulation equation with source terms, Quarterly of Applied Mathematics., LX (1), pp. 183-194, 2002.

[7] Laurençot, P., On a class of continuous coagulation-fragmentation equations, J. Diff. Eqns., 167, pp. 245-274, 2000. 
[8] Norris, J.R., Smoluchowski's coagulation equation: uniqueness, nonuniqueness and a hydrodynamic limit for the stochastic coalescent, The Annals of Applied Probability, 9(1), pp. 78-109, 1999.

[9] Wattis, J.A.D, McCartney, D.G. \& Gudmundsson, T., Coagulation equations with mass loss, Journal of Engineering Mathematics, 49, pp. 113-131, 2004.

[10] Calin, C.D., Shirvani, M. \& van Roessel, H.J., The coagulation equation with bounded kernels, and particle source and removal, to appear, AMI, Technical Reports, University of Alberta, 2006.

[11] Martynov, G.A. \& Bakanov, S.P., Solution of the kinetic equation for coagulation, In: B. Derjaguin, editor, Surface Forces, 1961.

[12] Filbet, F. \& Laurençot, P., Numerical simulation of the Smoluchowski coagulation equation, SIAM J. Sci. Comput., 25, pp. 2004-2028, 2004.

[13] Sandu, A., A framework for the numerical treatment of aerosol dynamics, Applied Numerical Mathematics, 45, pp. 475-497, 2003. 